\title{
Healthcare Costs of Smokers Using Varenicline Versus Nicotine-Replacement Therapy Patch in the United States: Evidence from Real-World Practice
}

\author{
Lauren J. Lee · Qian Li · Marianna Bruno · Birol Emir • \\ Brian Murphy · Surbhi Shah • Matthew Reynolds • Nick Marchant • \\ Peter W. Park
}

Received: November 20, 2018 / Published online: December 19, 2018

(C) The Author(s) 2018

\section{ABSTRACT}

Introduction: Varenicline (VAR) is an effective smoking-cessation therapy compared to the commonly used nicotine-replacement therapy patch (NRT-P). However, comparative realworld evidence on smoking-cessation therapies is limited, especially for economic outcomes.

Methods: Using national claims databases (2012-2016) in the United States (US), adults initiating VAR or NRT-P without use of any other smoking-cessation products were followed for up to 1 year on a quarterly basis. Outcomes included smoking-attributable (SA)

Enhanced Digital Features To view enhanced digital features for this article go to https://doi.org/10.6084/ m9.figshare.7415456.

Electronic supplementary material The online version of this article (https://doi.org/10.1007/s12325$018-0858-y)$ contains supplementary material, which is available to authorized users.

L. J. Lee $(\bowtie) \cdot$ N. Marchant

Health Economics and Outcomes Research, Pfizer, Inc., New York, NY, USA

e-mail: jiyoung.lee@pfizer.com

Q. Li · B. Murphy · S. Shah · M. Reynolds

Data Analytics, Evidera, Bethesda, MD, USA

M. Bruno - P. W. Park

Medical Affairs, Pfizer, Inc., New York, NY, USA

B. Emir

Statistical Research and Data Science Center, Pfizer, Inc., New York, NY, USA (cardiovascular, diabetes, pulmonary diseases, and smoking cessation) and all-cause costs (2017 US dollars). Adjusted mean costs were estimated from multivariable regressions, with baseline characteristics and propensity scores as covariates. Annual adjusted costs were calculated from quarterly averages.

Results: The VAR cohort $(n=209,284)$ was younger (mean age 46.7 vs. 49.0 years) and had fewer comorbidities [mean Charlson Comorbidity Index (CCI): 0.8 vs. 1.6$]$ than the NRT-P cohort $(n=34,593)$. After adjustment, VAR cohort had lower SA and all-cause medical costs than NRT-P cohort in Quarters 1-4 (Q1-Q4) of follow-up, and had lower SA and all-cause total costs in Q2-Q4. Annually, VAR cohort had higher SA total costs (\$307) and lower all-cause costs $(-\$ 2089)$ than NRT-P cohort. Annual medical costs were lower in VAR cohort $(-\$ 640$ for SA and - \$2876 for all-cause), and pharmacy costs were higher ( $\$ 762$ for SA and $\$ 777$ for allcause). In adherent patients (VAR: $n=38,744$; NRT-P: $n=2702)$, VAR patients had lower annual medical costs $(-\$ 794$ for SA and - \$1636 for all-cause) and higher pharmacy costs ( $\$ 1175$ for SA and $\$ 1269$ for all-cause); differences in SA and all-cause total costs were not statistically significant between treatment groups.

Conclusions: Lower SA and all-cause medical costs associated with the use of VAR versus NRT$P$ resulted in savings in all-cause total costs and, 
among adherent patients, potentially offset the high pharmacy costs of VAR.

Funding: Pfizer, Inc.

Keywords: Healthcare costs; Real-world evidence; Varenicline

\section{INTRODUCTION}

Cigarette smoking is the leading cause of preventable disease and death in the United States (US); it affects more than 16 million people and accounts for one in five deaths every year [1]. Although it has been estimated that current smoking declined from $20.9 \%$ in 2005 to $15.5 \%$ in 2016, the prevalence of smoking has not changed significantly [1]. In 2016, more than 15 out of 100 adults were current smokers [1]. Smoking affects nearly every organ of the body and results in a significant risk of developing heart disease, stroke, and lung cancer [2]. It is estimated that smoking increases the risk of coronary heart disease and stroke by two to four times, and the risk of lung cancer by 25 times in men and 25.7 times in women [2]. Smoking causes approximately $90 \%$ of all lung cancer deaths and $80 \%$ of all deaths from chronic obstructive pulmonary disease (COPD) $[3,4]$. It imposes a significant economic burden on society as well as due to increased healthcare resource use (HCRU) and absenteeism. Cigarette smoking accounts for more than US $\$ 300$ billion (2017 US dollars) each year in related illness, comprising approximately $\$ 170$ billion in direct medical care costs and $\$ 156$ billion in lost productivity $[2,5]$.

Smoking cessation has immediate and longterm health benefits. Even 3 months of not smoking can improve a person's health status, and quitting can reduce the risk for a heart attack within 1 year [6]. Based on US nationally representative databases, healthcare expenditures of former smokers are $70 \%$ lower than for current smokers [7], and improvement in work productivity was observed even in recent quitters [8]. Despite the substantial benefits and sufficient availability of smoking-cessation interventions, there was a modest increase in the prevalence of past-year quit attempts (from
49.2 to $55.4 \%$ ) and recent smoking cessation (from 5.7 to $7.4 \%$ ) in 2000-2015 [9, 10].

Varenicline (VAR; CHANTIX ${ }^{\circledR}$ ) is a nicotinic receptor partial agonist that was approved as an aid to smoking cessation in the US in May 2006; it is one of seven first-line medications recommended to treat tobacco use by the US Public Health Service guidelines [11]. In clinical trials, VAR has demonstrated greater efficacy than other single formulations of smoking-cessation therapies [12]. According to the 2016 Cochrane Systematic Review, the number needed to treat for one additional successful outcome was 11 for VAR [95\% confidence interval (CI): 9-13], 22 for bupropion (95\% CI 18-28), and 23 (95\% CI 20-25) for all types of nicotine replacement therapy (NRT), using various comparators available in the literature [13]. Studies on economic evaluations of smoking-cessation therapies have also demonstrated the superiority of VAR [15]. In a decision-analysis model of various US healthcare payers, use of VAR led to immediate healthcare cost savings compared with bupropion and savings within 2 years compared with nicotine patches or no aids [15].

In addition to the substantial evidence on the clinical efficacy of VAR, several populationbased health studies have assessed its real-world effectiveness [16-30]. The abstinence rate associated with VAR was moderately higher (approximately 30\%) than that of the NRT patch and marginally higher (approximately $8 \%$ ) than combination NRT within 6 months, based on data from national smoking-cessation programs in the United Kingdom (UK) and Taiwan $[31,32]$. In a recent, large, prospective cohort study of electronic medical records from 654 general practices in the UK, patients who initiated VAR were more likely to be abstinent up to 4 years than those initiating NRT [27]. Data on the comparative effectiveness of VAR versus other smoking-cessation therapies in the US were from specific smoking-cessation programs or local health centers. In the Montana Tobacco Quit Line program, participants using VAR $(n=3116)$ were more likely to be abstinent than those using NRT $(n=3697)$ at 3 and 6 months of treatment [16]. Other studies in the US, based on smaller study samples $(n<1000)$ have found no difference in the abstinence rate associated 
with VAR versus other smoking-cessation therapies [19, 25].

The translation of clinical differences among smoking-cessation therapies on HCRU and costs has yet to be addressed in the literature. Some studies assessed the impact of smoking cessation in terms of hospitalization and/or mortality $[18,33,34]$. For example, the risk of hospitalization due to acute myocardial infarction and other acute coronary heart disease diagnoses fell after use of an NRT product or VAR among Massachusetts Medicaid enrollees [35]. To our knowledge, only a few studies have examined healthcare costs as outcome measures in smoking cessation [36, 37]. Using data from the Medical University of South Carolina, a recent study found that charges for hospitalization, emergency room (ER), and ambulatory surgery visits of patients receiving a Tobacco Dependence Treatment Service (TDTS) were $\$ 7299$ lower during the year after admission compared to those for patients without TDTS [36]. In a group of patients with COPD who were treated in routine clinical practice in Spain, current smokers were found to have significantly higher HCRU and costs than former smokers [37].

The economic impact of smoking-cessation therapies has yet to be evaluated via comparative analysis in real-world practices in the US. Thus, the objective of this study was to compare smoking-attributable (SA) and all-cause healthcare costs among smokers who were prescribed VAR versus a commonly used NRT patch in the US using a national administrative claims dataset.

\section{METHODS}

\section{Data Source}

The Truven Health MarketScan ${ }^{\circledR}$ Commercial Claims and Encounters and Medicare Supplemental databases were used. The combined dataset contains administrative claims and eligibility records of approximately 30 million commercially insured individuals and their dependents, as well as approximately 3 million enrollees in Medicare supplemental plans each year in the US. Besides patient information on age, gender, geographical region, health insurance payer type, employment status, and monthly enrollment status, the data contain detailed information on medical claims (i.e., inpatient and outpatient), including but not limited to date and duration of services, provider type, payments, and pharmacy claims, including but not limited to quantities supplied and paid amounts of drugs. All databases are Health Insurance Portability and Accountability Act compliant. Since this study utilized deidentified administrative claims, institutional review board approval was not required.

\section{Study Design and Sample Selection}

A retrospective cohort study with intent-to-treat design was implemented. Patients with pharmacy claims for VAR or NRT patch from January 2012 through December 2016 were identified. The first pharmacy claim defined the index therapy (VAR cohort or NRT patch cohort) and the index date. In this study, the smoking-cessation therapies were those prescribed in outpatient settings, unless otherwise specified.

The baseline period covered 6 months before the index date, and data in the follow-up period were examined on a quarterly basis up to 1 year or the end of continuous enrollment in health plans, whichever came first (Fig. 1). Patients were not required to have at least 1 year of follow-up to avoid potential implications from immortal time bias (i.e., only patients with long survival were included in the study).

Patients were included in the study if they were at least 18 years old at the index date, had continuous enrollment in health plans (medical and pharmacy) from 6 months before to 3 months after the index date; and had positive days supplied for VAR or NRT patch (but not both) at the index date. Patients were further required to only use their index therapy, without pharmacy claims for any other smokingcessation therapies (including bupropion and NRT gum, inhaler, lozenge, or nasal spray) in baseline or follow-up periods. The resulting study sample was referred to as the "initiator population". An "adherent population" of VAR 


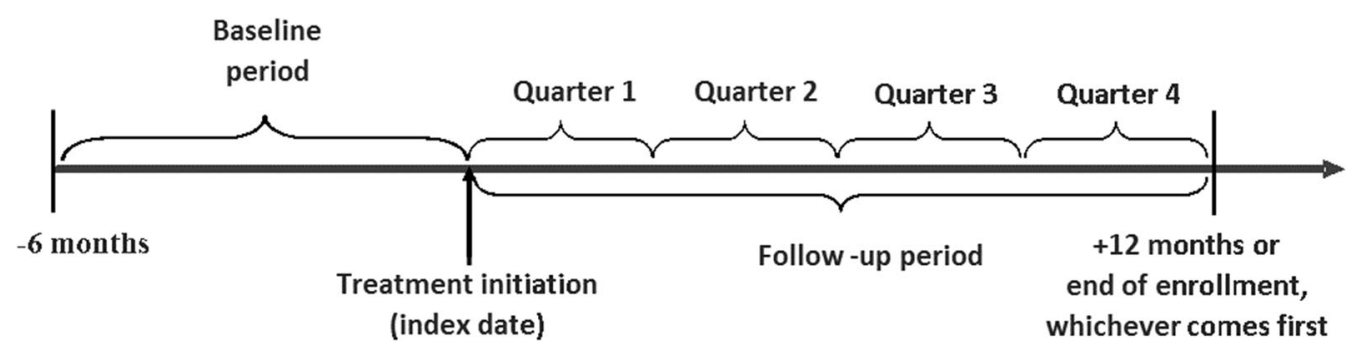

Fig. 1 Study periods

or NRT patch users was further identified if patients had a medication possession ratio of the index therapy (calculated as total days supplied divided by 90 days) of at least $80 \%$ in the first quarter of follow-up.

\section{Study Measures}

Patient characteristics were assessed during the baseline period or as of the index date. Demographic characteristics at the index date included age, gender, geographic region, residential setting, health plan type, and year of therapy initiation. Clinical characteristics assessed during the baseline period included Charlson Comorbidity Index (CCI) [38], diagnosis of specific conditions (as listed in Table 1), use of smoking-cessation counseling, and occurrence of hospitalization. The healthcare costs incurred during the baseline period were also examined.

The study outcomes were SA and all-cause healthcare costs incurred during each quarter of the defined follow-up period. Total all-cause costs included all medical and pharmacy claims paid by patients and commercial insurers. Allcause medical costs were further stratified by healthcare settings of outpatient, inpatient, or ER services.

SA healthcare costs included medical and pharmacy costs to treat SA conditions and costs for smoking cessation. The SA conditions, adapted from the 2014 Surgeon General's Report on the health consequences of smoking [2], included cardiovascular diseases (heart disease: coronary, pulmonary, rheumatic, and other; arterial disease: cerebrovascular, atherosclerosis, aortic aneurysm, and other), diabetes mellitus, and pulmonary conditions (acute conditions of influenza, pneumonia, tuberculous, and COPD). Total SA costs included SA medical costs (which covered inpatient claims with a primary diagnosis of an SA condition, outpatient and ER claims with any diagnosis of an SA condition, and medical claims for smoking counseling visits), and SA pharmacy costs [which covered smoking-cessation therapies (i.e., index therapy) and treatments for SA conditions].

All conditions and specific services were identified through International Classification of Diseases, Ninth Revision, Clinical Modification (ICD-9-CM), International Classification of Diseases, 10th Revision, Clinical Modification (ICD-10-CM), Current Procedural Terminology (CPT), and Healthcare Common Procedure Coding System (HCPCS) codes (listed in Table S1). Costs were adjusted to 2017 dollars using the US Medical Care Consumer Price Index.

\section{Statistical Analysis}

The study measures were compared between cohorts using Student's $t$ test for continuous variables and the Chi square test for categorical variables. The association between healthcare costs in each quarter of follow-up and index therapy was assessed by multivariable regression models. Nearly $10 \%$ or $>10 \%$ of quarters in each cohort had patients with zero total allcause or SA costs, respectively. Thus a two-part model, which has been commonly used to handle overabundance of zero values, was implemented [39]. Specifically, costs were modeled as $E(y)=\operatorname{prob}(y>0) \times E(y \mid y>0)+$ $\operatorname{prob}(y=0) \times 0=\operatorname{prob}(y>0) \times \mathrm{E}(y \mid y>0)$, with $E(y)$ as expected costs, $\operatorname{prob}(y>0)$ as probability of incurring any costs, and $E(y \mid y>0)$ as 
Table 1 Patient characteristics of the initiator and adherent study populations

\begin{tabular}{|c|c|c|c|c|}
\hline \multirow[t]{2}{*}{ Patient characteristics ${ }^{\mathbf{a}}$} & \multicolumn{2}{|c|}{ Initiator population } & \multicolumn{2}{|c|}{ Adherent population } \\
\hline & $\begin{array}{l}\text { Varenicline } \\
(n=209,284)\end{array}$ & $\begin{array}{l}\text { NRT patch } \\
(n=34,593)\end{array}$ & $\begin{array}{l}\text { Varenicline } \\
(n=38,744)\end{array}$ & $\begin{array}{l}\text { NRT patch } \\
(n=2702)\end{array}$ \\
\hline Age & 46.7 (11.9) & $49.0(13.1)$ & $48.9(11.1)$ & $51.3(12.2)$ \\
\hline Male & $106,103(50.7 \%)$ & $16,557(47.9 \%)$ & $19,930(51.4 \%)$ & $1385(51.3 \%)^{N S}$ \\
\hline \multicolumn{5}{|c|}{ Health plan out-of-network coverage } \\
\hline Covered & $21,242(10.1 \%)$ & $5833(16.9 \%)$ & $3669(9.5 \%)$ & $369(13.7 \%)$ \\
\hline Not covered & $145,127(69.3 \%)$ & $21,931(63.4 \%)$ & $26,699(68.9 \%)$ & $1759(65.1 \%)$ \\
\hline Other/missing & $42,915(20.5 \%)$ & $6829(19.7 \%)$ & $8376(21.6 \%)$ & $574(21.2 \%)$ \\
\hline \multicolumn{5}{|l|}{ Region } \\
\hline Northeast & $37,621(18.0 \%)$ & $9650(27.9 \%)$ & $6950(17.9 \%)$ & $783(29.0 \%)$ \\
\hline North Central & $59,193(28.3 \%)$ & $10,248(29.6 \%)$ & $11,034(28.5 \%)$ & $821(30.4 \%)$ \\
\hline South & $81,008(38.7 \%)$ & $9732(28.1 \%)$ & $14,752(38.1 \%)$ & $708(26.2 \%)$ \\
\hline West & $28,534(13.6 \%)$ & $4626(13.4 \%)$ & $5527(14.3 \%)$ & $365(13.5 \%)$ \\
\hline Unknown/missing & $2928(1.4 \%)$ & $337(1.0 \%)$ & $481(1.2 \%)$ & $25(0.9 \%)$ \\
\hline Residence type, urban & $173,208(82.8 \%)$ & $28,329(81.9 \%)$ & $32,306(83.4 \%)$ & $2206(81.6 \%)$ \\
\hline \multicolumn{5}{|l|}{ Index year } \\
\hline 2012 & $35,692(17.1 \%)$ & $2674(7.7 \%)$ & $6165(15.9 \%)$ & $214(7.9 \%)$ \\
\hline 2013 & $57,631(27.5 \%)$ & $5838(16.9 \%)$ & $9874(25.5 \%)$ & $420(15.5 \%)$ \\
\hline 2014 & $48,454(23.2 \%)$ & $7918(22.9 \%)$ & $8923(23.0 \%)$ & $627(23.2 \%)$ \\
\hline 2015 & $37,392(17.9 \%)$ & $9466(27.4 \%)$ & $7296(18.8 \%)$ & $764(28.3 \%)$ \\
\hline 2016 & $30,115(14.4 \%)$ & $8697(25.1 \%)$ & $6486(16.7 \%)$ & $677(25.1 \%)$ \\
\hline \multicolumn{5}{|l|}{ Specific conditions } \\
\hline Tobacco dependence & $50,117(23.9 \%)$ & $13,353(38.6 \%)$ & $9413(24.3 \%)$ & $1017(37.6 \%)$ \\
\hline SA conditions & $71,030(33.9 \%)$ & $19,877(57.5 \%)$ & $14,412(37.2 \%)$ & $1575(58.3 \%)$ \\
\hline Cardiovascular diseases & $21,736(10.4 \%)$ & $7716(22.3 \%)$ & $4673(12.1 \%)$ & $647(23.9 \%)$ \\
\hline Diabetes mellitus & $18,057(8.6 \%)$ & $4514(13.0 \%)$ & $4141(10.7 \%)$ & $377(14.0 \%)$ \\
\hline Pulmonary conditions & $20,990(10.0 \%)$ & $6340(18.3 \%)$ & $4260(11.0 \%)$ & $531(19.7 \%)$ \\
\hline $\begin{array}{l}\text { Neuropsychiatric } \\
\text { conditions }\end{array}$ & $32,109(15.3 \%)$ & $10,838(31.3 \%)$ & $6128(15.8 \%)$ & $795(29.4 \%)$ \\
\hline $\begin{array}{l}\text { Cancer (excluding skin } \\
\text { cancer) }\end{array}$ & $5104(2.4 \%)$ & $1678(4.9 \%)$ & $1075(2.8 \%)$ & $137(5.1 \%)$ \\
\hline CCI & $0.8(1.3)$ & $1.6(2.1)$ & $0.9(1.4)$ & $1.5(2.0)$ \\
\hline
\end{tabular}


Table 1 continued

\begin{tabular}{|c|c|c|c|c|}
\hline \multirow[t]{2}{*}{ Patient characteristics ${ }^{\mathrm{a}}$} & \multicolumn{2}{|c|}{ Initiator population } & \multicolumn{2}{|c|}{ Adherent population } \\
\hline & $\begin{array}{l}\text { Varenicline } \\
(n=209,284)\end{array}$ & $\begin{array}{l}\text { NRT patch } \\
(n=34,593)\end{array}$ & $\begin{array}{l}\text { Varenicline } \\
(n=38,744)\end{array}$ & $\begin{array}{l}\text { NRT patch } \\
(n=2702)\end{array}$ \\
\hline $\begin{array}{l}\text { Any smoking-cessation } \\
\text { counseling }\end{array}$ & $9842(4.7 \%)$ & $2116(6.1 \%)$ & $1910(4.9 \%)$ & $176(6.5 \%)$ \\
\hline Any SA hospitalization & $2694(1.3 \%)$ & $3290(9.5 \%)$ & $623(1.6 \%)$ & $273(10.1 \%)$ \\
\hline $\begin{array}{l}\text { Any all-cause } \\
\text { hospitalization }\end{array}$ & $8140(3.9 \%)$ & $8851(25.6 \%)$ & $1621(4.2 \%)$ & $615(22.8 \%)$ \\
\hline \multicolumn{5}{|l|}{ SA costs } \\
\hline Total costs & $\$ 1226(\$ 7030)$ & $\$ 5519(\$ 20,586)$ & $\$ 1564(\$ 7922)$ & $\$ 6950(\$ 30,770)$ \\
\hline Medical costs & $\$ 936(\$ 6856)$ & $\$ 5133(\$ 20,444)$ & $\$ 1170(\$ 7709)$ & $\$ 6466(\$ 30,664)$ \\
\hline Outpatient costs & $\$ 403(\$ 3333)$ & $\$ 980(\$ 5406)$ & $\$ 486(\$ 3393)$ & $\$ 1126(\$ 7091)$ \\
\hline Inpatient/ER costs & $\$ 532(\$ 5467)$ & $\$ 4153(\$ 18,891)$ & $\$ 684(\$ 6248)$ & $\$ 5339(\$ 29,335)$ \\
\hline Pharmacy costs & $\$ 290(\$ 945)$ & $\$ 386(\$ 1238)$ & $\$ 394(\$ 1162)$ & $\$ 485(\$ 1371)$ \\
\hline \multicolumn{5}{|l|}{ All-cause costs } \\
\hline Total costs & $\$ 5281(\$ 15,141)$ & $\$ 17,421(\$ 38,099)$ & $\$ 5927(\$ 15,598)$ & $\$ 18,442(\$ 48,293)$ \\
\hline Medical costs & $\$ 4162(\$ 14,131)$ & $\$ 15,893(\$ 37,230)$ & $\$ 4580(\$ 14,678)$ & $\$ 16,588(\$ 47,639)$ \\
\hline Outpatient costs & $\$ 2536(\$ 8695)$ & $\$ 4722(\$ 13,859)$ & $\$ 2742(\$ 8217)$ & $\$ 4451(\$ 12,075)$ \\
\hline Inpatient/ER costs & $\$ 1625(\$ 9702)$ & $\$ 11,171(\$ 31,858)$ & $\$ 1839(\$ 10,668)$ & $\$ 12,138(\$ 44,238)$ \\
\hline Pharmacy costs & $\$ 1119(\$ 4222)$ & $\$ 1528(\$ 5872)$ & $\$ 1347(\$ 4075)$ & $\$ 1853(\$ 5722)$ \\
\hline $\begin{array}{l}\text { Number of quarters in } \\
\text { follow-up }\end{array}$ & $3.2(1.2)$ & $3.0(1.2)$ & $3.2(1.2)$ & $3.0(1.2)$ \\
\hline
\end{tabular}

$N S$ indicates $p>0.05$; all differences between VAR and NRT patch cohort were statistically significant at 0.05 level, unless noted otherwise

$C C I$ Charlson Comorbidity Index, ER emergency room, NRT nicotine-replacement therapy, $N S$ not significant, $S A$ smoking-attributable

a $n(\%)$ were reported for categorical/binary variables, and mean (standard deviation) were reported for continuous variables

expected costs given any costs. In this study, the model was specified as a logistic regression on the probability of incurring any costs in a quarter and, among quarters with any costs incurred, a generalized linear model (with gamma distribution and log link) on quarterly costs. In each regression of the two-part model, the generalized estimation equation (GEE) technique was implemented to the entire study sample to control for correlations between outcomes in multiple quarters of a patient [40].
All regressions were modeled as a function of interactions between index therapy and sequential quarter number [i.e., VAR vs. NRT patch with follow-up in Quarter $1(\mathrm{Q} 1)$, Quarter 2 (Q2), Quarter 3 (Q3), and Quarter 4 (Q4), respectively] and were adjusted for the following baseline characteristics: demographics, CCI, diagnosis of specific conditions [cardiovascular diseases, diabetes mellitus, pulmonary conditions, neuropsychiatric conditions, malignancy (excluding skin cancer), and tobacco dependence], use of smoking-cessation counseling, 
occurrence of hospitalization (SA or all-cause, corresponding to study outcomes), and healthcare costs corresponding to study outcomes (e.g., baseline SA medical costs were included as covariate for regressions on quarterly SA medical costs in follow-up). Propensity scores (i.e., the predicted probability to be treated by the index therapy) were also included in the regressions as one covariate. These scores were estimated using logistic regression, with the index therapy as the outcome variable, adjusting for baseline variables (as listed in Table S3). The number of quarters in follow-up was also considered in the propensity score estimation in an attempt to balance the duration of follow-up between cohorts.

Based on the regression results, the adjusted costs associated with the use of VAR or NRT patch in each quarter of follow-up were calculated by assuming all patients with enrollment in the quarter were in the VAR or NRT patch cohort, respectively. The differences in mean adjusted costs per patient in each quarter were calculated, and the 95\% confidence intervals (CIs) were estimated using 1000 times of biascorrected bootstraps [39]. The quarterly averages per patient were calculated as the sum of costs over the follow-up period of all patients as if they were in the VAR or NRT patch cohort, respectively, divided by the sum of the followup duration (in quarters) of all patients. The quarterly averages then were multiplied by four to estimate the average costs the patients would incur if they had 1 year of follow-up (i.e., adjusted annual costs) and differences between cohorts. The analyses were applied to both the initiator and adherent populations. All analyses were conducted using SAS v.9.4 (SAS Institute, Cary, NC, USA).

\section{Sensitivity Analysis}

To assess the robustness of study results, sensitivity analyses were conducted by applying propensity score matching. Due to the sample size, patients in the VAR cohort were matched to patients in the NRT patch cohort using a 2:1 ratio. Similar patient characteristics, as considered in the main analysis, were used to estimate the propensity scores (Table S3). The balance between cohorts after matching was assessed by standardized difference, which was calculated as the mean or proportion difference divided by the pooled standard deviation. A standardized difference $<10 \%$ has been understood to indicate a negligible difference in the mean or prevalence of a covariate between cohorts [41]. The healthcare costs, annualized for each patient, were compared between matched cohorts. This approach was also applied to a subgroup of patients with at least 12 months of follow-up.

\section{Compliance with Ethics Guidelines}

This article does not contain any studies with human participants or animals performed by any of the authors.

\section{RESULTS}

\section{Patient Characteristics}

The study sample included 209,284 VAR initiators and 34,593 NRT patch initiators who met the sample selection criteria (Fig. 2). Among them, the adherent population was identified in $18.5 \%$ $(n=38,744)$ of the VAR cohort and $7.8 \%$ $(n=2702)$ of the NRT patch cohort. The patient characteristics of the two cohorts differed significantly in many aspects (Table 1). In the initiator population, patients in the VAR cohort were younger [mean age (standard deviation) 46.7 (11.9) vs. 49.0 (13.1) years, $p<0.05$ ]; more likely to be males ( $50.7 \%$ vs. $47.9 \%)$; to have a health plan without out-of-network coverage $(69.3 \%$ vs. $63.4 \%)$; and to be from the south $(38.7 \%$ vs. $28.1 \%$, all $p<0.05)$. The VAR cohort also had longer follow-up compared with the NRT patch cohort (mean quarters 3.2 vs. 3.0, $p<0.05$ ).

Patients in the VAR cohort had fewer comorbidities than those in the NRT patch cohort. During baseline for the initiator population, the VAR cohort had a lower CCI and lower prevalence of any SA conditions, tobacco dependence, neuropsychiatric (NPS) conditions, and cancer than the NRT patch cohort (Table 1 ; all $p<0.05$ ). VAR 


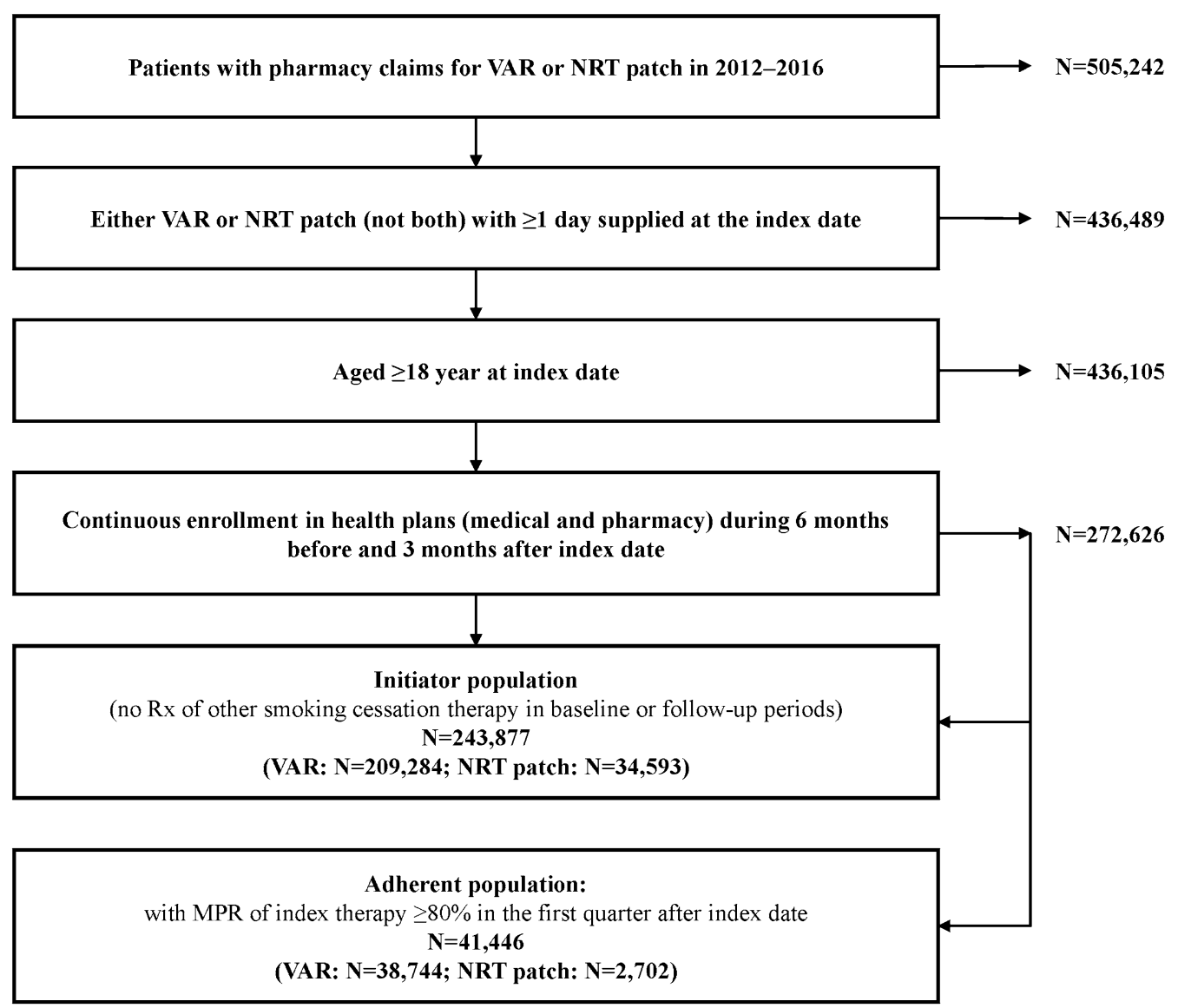

Fig. 2 Sample selection of patients in initiator and adherent populations. $N R T$ nicotine-replacement therapy, $R X$ prescription, $M P R$ medication possession ratio, VAR varenicline

initiators also had lower baseline HCRU (including any use of smoking-cessation counseling, and any SA and all-cause hospitalization) and healthcare costs (including SA and all-cause total costs, and all cost categories) than NRT patch initiators (all $p<0.05$ ).

No noticeable differences in patient characteristics were observed between the adherent and initiator populations. The differences between the VAR and NRT cohorts in the initiator population were also observed in the adherent population, except for the gender distribution, which was similar between cohorts of adherent users.

\section{Healthcare Costs during Follow-up}

The quarterly healthcare costs by setting without any adjustment are summarized in Table S2, and the regression coefficients on treatment cohort (VAR vs. NRT patch) in the two-part models are presented in Table S4. The mean differences in adjusted costs between the VAR cohort versus the NRT patch cohort in each quarter during follow-up are illustrated in Fig. 3. Similar trends over time were demonstrated in the initiator and adherent populations, although with varying statistical significancethe VAR cohort had lower medical costs (SA and all-cause) than the NRT patch cohort throughout the year, especially in Q2-Q4; had significantly higher pharmacy costs in Q1; and had higher total costs in Q1 but lower total costs in Q2-Q4. These results signal early economic benefits of VAR over the NRT patch in medical cost offset.

In the initiator population, the SA medical costs in the VAR cohort were lower than those 


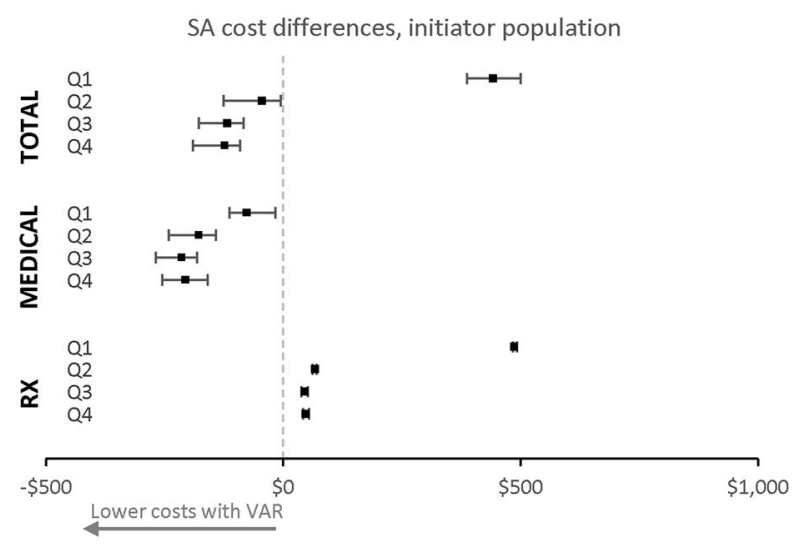

SA cost differences, adherent population

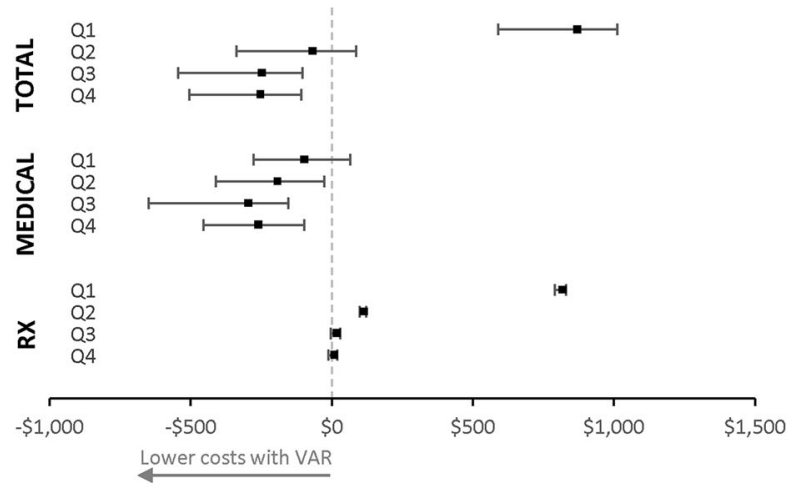

Fig. 3 Adjusted cost differences (varenicline vs. NRT patch) by quarter. Adjusted differences between the VAR and NRT patch cohorts and the $95 \% \mathrm{CI}$ are presented; adjusted costs in each quarter were estimated from multivariable regression models, adjusting for the following baseline characteristics-demographics, CCI, diagnosis of specific conditions [cardiovascular diseases, diabetes

for the NRT patch cohort in Q1-Q4 (all $p<0.05)$; SA pharmacy costs were higher in the VAR cohort throughout the year (all $p<0.05$ ), especially in Q1 (Fig. 3). These trends led to SA total costs among initiators of VAR being higher than those for NRT patch in Q1 but lower in Q2-Q4 (all $p<0.05$ ). The VAR initiators also had lower all-cause medical costs than NRT initiators throughout the year (Q1-Q4, all $p<0.05)$, but their pharmacy costs were higher in Q1 $(p<0.05)$, with no significant differences between cohorts in Q2-Q4. As a result, the allcause total costs were lower in the VAR cohort than the NRT patch cohort in Q2-Q4, with no difference between cohorts in Q1. While, in the adherent population, the VAR cohort had lower

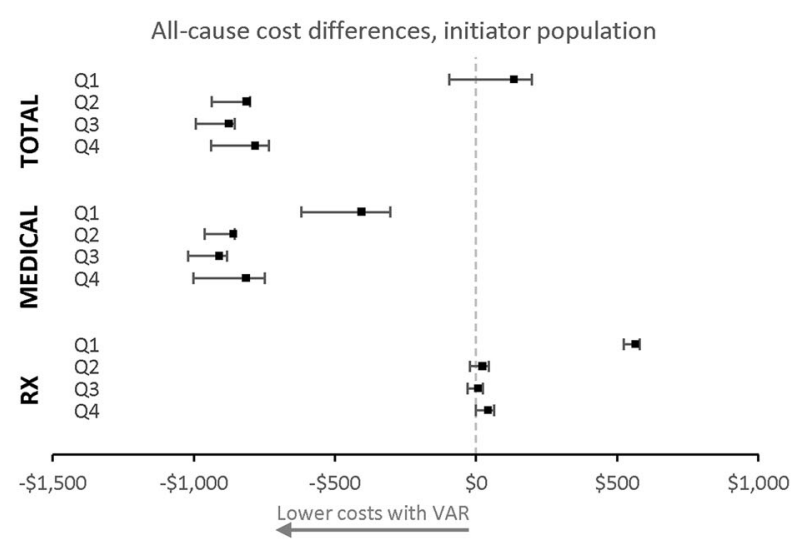

All-cause cost differences, adherent population

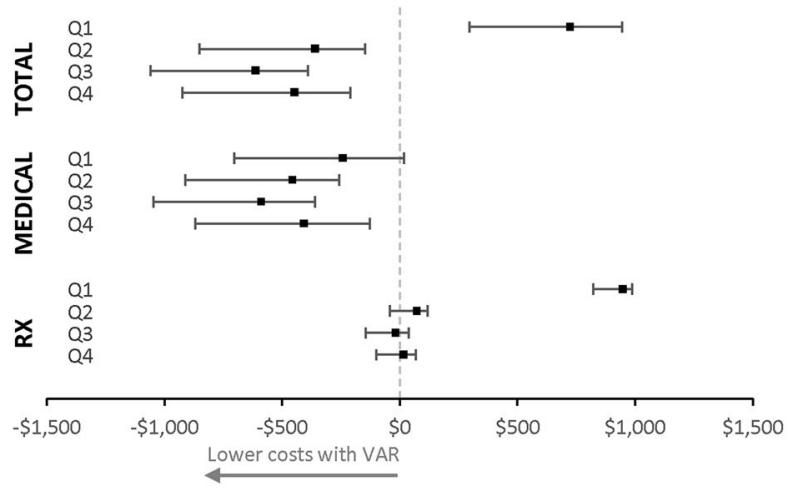

mellitus, pulmonary conditions, neuropsychiatric conditions, malignancy (excluding skin cancer), and tobacco dependence], use of smoking-cessation counseling, occurrence of hospitalization (SA or all cause, corresponding to study outcomes), healthcare costs (corresponding to study outcomes)-and propensity scores. $R X$ pharmacy, $S A$ smoking-attributable, $V A R$ varenicline

medical costs (SA and all-cause) in Q2-Q4 and higher all-cause total costs in Q1 (all $p<0.05$ ) than the NRT patch cohort.

The annual adjusted costs and differences between cohorts, estimated from the quarterly average per patient per year, are reported in Fig. 4. Use of VAR, as compared to the NRT patch, was associated with $\$ 640$ (or 23\%) lower SA medical costs (\$2164 vs. \$2803) and \$2876 $(21 \%)$ lower all-cause medical costs $(\$ 11,049$ vs. $\$ 13,925$ ) during the first year of initiation (all $p<0.05)$. The savings in medical costs associated with use of VAR versus the NRT patch were reflected by lower annual outpatient costs [ $\$ 210$ (16\%) lower for SA and \$1505 (19\%) lower for all-cause] and inpatient/ER costs [\$471 (31\%) 
Annual SA costs, initiator population

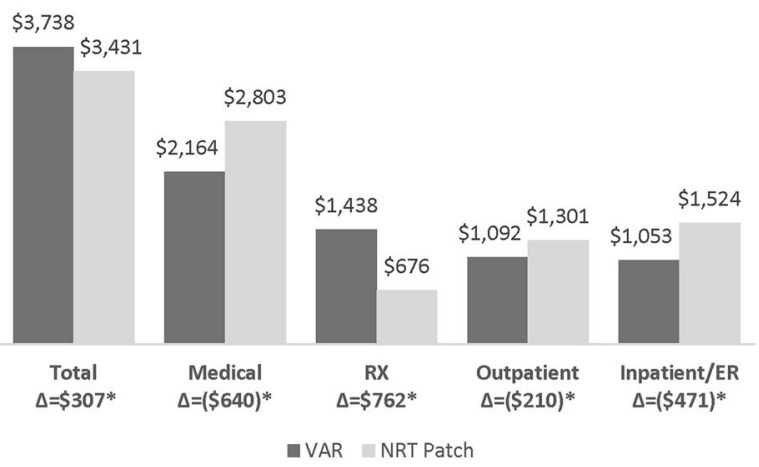

Annual SA costs, adherent population

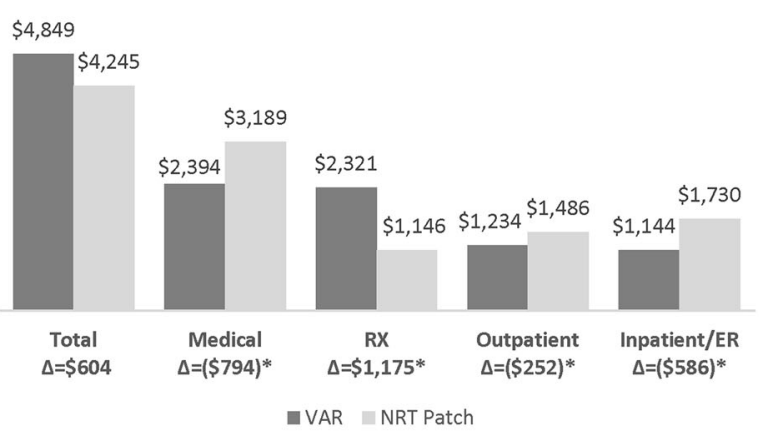

Fig. 4 Adjusted annual costs. ${ }^{*} P$ value $<0.05$; difference between cohorts may not be exact due to rounding; adjusted annual costs, estimated as four times the quarterly average of adjusted costs per patient, showed the average costs would incur if the patients had 1 year of follow-up; adjusted costs in each quarter were estimated from multivariable regression models, adjusting for the following baseline characteristics-demographics, CCI, diagnosis of specific conditions [cardiovascular diseases, diabetes

lower for SA and \$1538 (25\%) lower for allcause, all $p<0.05]$. The annual pharmacy costs were higher in the VAR cohort than the NRT patch cohort [\$762 (113\%) higher for SA and $\$ 777$ or (32\%) higher for all-cause, all $p<0.05]$. The resulting total SA healthcare costs were $\$ 307$ (9\%) higher annually (\$3738 vs. \$3431) for VAR users compared with NRT patch users, but total all-cause healthcare costs were $\$ 2089$ $(13 \%)$ lower annually $(\$ 14,486$ vs. $\$ 16,576$, all $p<0.05)$. In the adherent population, use of VAR versus the NRT patch was also associated with savings in medical costs annually [ $\$ 794$ (25\%) lower for SA and \$1636 (13\%) lower for all-cause] and higher pharmacy costs (all

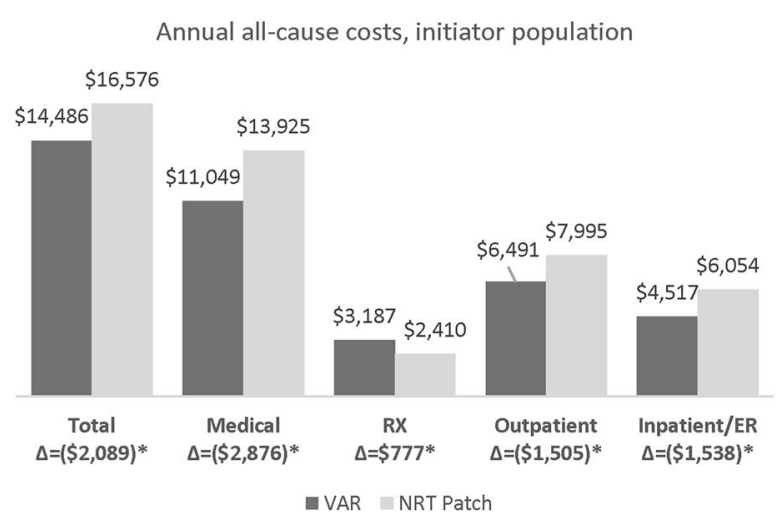

Annual all-cause costs, adherent population

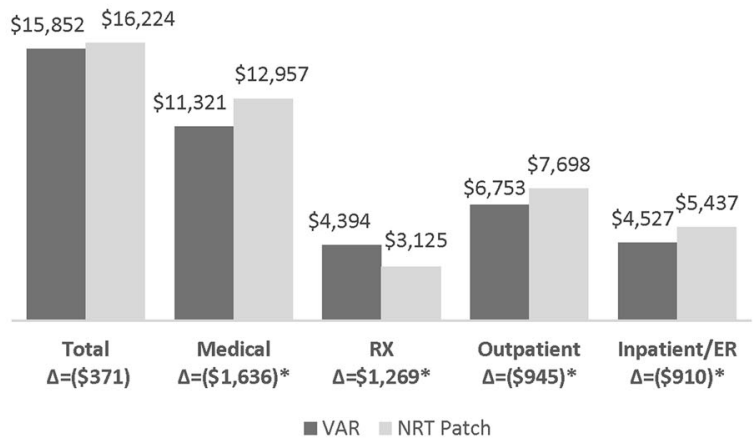

mellitus, pulmonary conditions, neuropsychiatric conditions, malignancy (excluding skin cancer), and tobacco dependence], use of smoking-cessation counseling, occurrence of hospitalization (SA or all cause, corresponding to study outcomes), healthcare costs (corresponding to study outcomes)—and propensity scores. ER emergency room, $N R T$ nicotine replacement therapy, $R X$ pharmacy, $S A$ smoking-attributable, VAR varenicline

$p<0.05)$. The differences in annual total SA and all-cause healthcare costs between cohorts of adherent users were not statistically significant.

\section{Sensitivity Analysis}

All propensity score-matched cohorts were balanced as measured by $<10 \%$ of standardized differences in patient characteristics (Table S5). Compared to NRT patch patients $(n=26,550)$, the matched VAR patients $(n=53,100)$ had lower annualized all-cause medical $(\$ 13,045$ vs. $\$ 15,583)$ and total costs $(\$ 17,375$ vs. $\$ 18,688$, 
Table 2 Sensitivity analysis: annual costs in follow-up after propensity score matching

\begin{tabular}{|c|c|c|c|c|}
\hline \multirow[b]{2}{*}{$\begin{array}{l}\begin{array}{l}\text { Initiator } \\
\text { population }\end{array} \\
\end{array}$} & \multicolumn{2}{|c|}{$\begin{array}{l}\text { Study sample in main analysis } \\
\text { after matching }\end{array}$} & \multicolumn{2}{|c|}{$\begin{array}{l}\text { Subgroup with } \geq 12 \text { months of follow-up } \\
\text { after matching }\end{array}$} \\
\hline & $\begin{array}{l}\text { Varenicline } \\
(n=53,100)\end{array}$ & $\begin{array}{l}\text { NRT Patch } \\
(n=26,550)\end{array}$ & $\begin{array}{l}\text { Varenicline } \\
(n=29,408)\end{array}$ & $\begin{array}{l}\text { NRT Patch } \\
(n=14,704)\end{array}$ \\
\hline SA total costs & $\$ 4433(\$ 21,710)$ & $\$ 3891(\$ 19,085)^{*}$ & $\$ 3998(\$ 15,535)$ & $\$ 3894(\$ 15,622)$ \\
\hline SA medical costs & $\$ 2598(\$ 21,281)$ & $\$ 2990(\$ 18,699)^{*}$ & $\$ 2511(\$ 14,999)$ & $\$ 2998(\$ 15,187)^{*}$ \\
\hline All-cause total costs & $\$ 17,375(\$ 48,677)$ & $\$ 18,688(\$ 58,133)^{*}$ & $\$ 16,207(\$ 37,872)$ & $\$ 17,861(\$ 46,277)^{*}$ \\
\hline All-cause medical costs & $\$ 13,045(\$ 46,512)$ & $\$ 15,583(\$ 55,979)^{*}$ & $\$ 12,218(\$ 34,761)$ & $\$ 14,577(\$ 43,345)^{*}$ \\
\hline $\begin{array}{l}\text { Adherent } \\
\text { population }\end{array}$ & $\begin{array}{l}\text { Varenicline } \\
(n=5048)\end{array}$ & $\begin{array}{l}\text { NRT patch } \\
(n=2524)\end{array}$ & $\begin{array}{l}\text { Varenicline } \\
(n=2104)\end{array}$ & $\begin{array}{l}\text { NRT patch } \\
(n=1052)\end{array}$ \\
\hline SA total costs & $\$ 7506(\$ 26,296)$ & $\$ 7014(\$ 32,067)$ & $\$ 5234(\$ 20,042)$ & $\$ 3863(\$ 14,427)^{*}$ \\
\hline SA medical costs & $\$ 4285(\$ 25,494)$ & $\$ 5439(\$ 31,566)$ & $\$ 2979(\$ 19,285)$ & $\$ 2659(\$ 14,020)$ \\
\hline All-cause total costs & $\$ 23,417(\$ 60,732)$ & $\$ 25,491(\$ 66,265)$ & $\$ 17,560(\$ 35,943)$ & $\$ 15,346(\$ 30,563)$ \\
\hline All-cause medical costs & $\$ 17,265(\$ 55,540)$ & $\$ 20,910(\$ 64,123)^{*}$ & $\$ 12,209(\$ 32,152)$ & $\$ 11,533(\$ 27,590)$ \\
\hline
\end{tabular}

Mean (standard deviation) were reported for continuous variables

$N R T$ nicotine replacement therapy, $S A$ smoking-attributable

${ }^{*} p$ value $<0.05$

all $p<0.05$ ) (Table 2). The annualized SA medical costs were lower ( $\$ 2598$ vs. $\$ 2990)$, but the annualized SA total costs were higher in the matched VAR cohort versus the NRT patch cohort ( $\$ 4433$ vs. $\$ 3891$, all $p<0.05$ ). Similar results were found among matched cohorts with at least 12 months of follow-up (VAR: $n=29,408$; NRT patch: $n=14,704)$, while the difference in SA total costs between cohorts was not statistically significant. In the adherent population, the VAR cohort $(n=5048)$, as compared to the matched NRT patch cohort $(n=2524)$, had lower annualized all-cause medical costs $(\$ 17,265$ vs. $\$ 20,910)$; among those with at least 12 months of follow-up (VAR: $n=2104$; NRT patch: $n=1052$ ), the VAR cohort had higher annualized SA total costs (\$5234 vs. \$3863, all $p<0.05$ ).

\section{DISCUSSION}

To our knowledge, this is the first comparative analysis exploring the economic impact of using VAR versus NRT patch in US general practices. After adjusting for differences in patient characteristics between cohorts, VAR initiators had lower medical costs (SA and allcause) than NRT patch initiators throughout the year, especially in Q2-Q4; however, their pharmacy costs were noticeably higher in Q1. Overall, use of VAR versus the NRT patch was associated with lower [\$2089 (13\%)] all-cause total healthcare costs but higher [\$307 (9\%)] SA total costs during the year after initiation. Among adherent users, the differences in annual total costs (SA and all-cause) between cohorts were not statistically significant.

Assessing the comparative economic outcomes associated with the use of different pharmacotherapies is important in decisionmaking for healthcare providers, as well as for private and public healthcare payers. Although VAR has demonstrated real-world effectiveness compared with NRT, the costs of VAR were much higher (1-month supply was around $\$ 300$ for VAR and around $\$ 30$ for the NRT patch). 
Thus, there was a need to investigate the cost impact of using VAR in general practices in the US. Our study provided evidence that smokers using VAR incurred lower healthcare costs than those using the NRT patch. Further research is warranted to assess the economic impact of VAR from different perspectives.

Our study not only estimated the overall economic impact of using VAR during the year after therapy initiation but also demonstrated how the impact changed over time. Savings in medical costs associated with the use of VAR versus the NRT patch were found after the first 3 months following therapy initiation. This is consistent with the effectiveness of VAR found in clinical trials and population studies. In the Evaluating Adverse Events in a Global Smoking Cessation Study (EAGLES), the largest clinical trial on smoking-cessation treatments $(n=8144$ smokers), patients treated with VAR achieved higher abstinence rates at week 12 than those on a nicotine patch, bupropion, or placebo [42]. Based on data from a smoking-cessation program in the US, smokers using VAR compared with NRT were more likely to be abstinent at 3 and 6 months after selecting the therapy. In our study, savings in medical costs generally offset the high pharmacy costs in the VAR cohort, which was reflected by similar or lower total costs associated with the use of VAR versus the NRT patch; this observation remained stable from Q2 to the end of 1 year of follow-up. This finding supports an opportunity to further evaluate the longer-term economic impact associated with initiating and using smokingcessation pharmacotherapies.

The overall impact during the year after therapy initiation varied based on level of adherence to the index therapies. VAR initiators, regardless of their adherence level to index therapy, had higher annual SA total costs than NRT patch initiators, despite their savings in medical costs; however, they had lower annual all-cause total costs. Among patients with adherent use of index therapies, the differences in SA and all-cause total costs were not statistically significant between cohorts. A published study has shown that the effectiveness of VAR is associated with medication adherence. Liberman et al. examined smoking status among
VAR initiators from a regional healthcare system in the US [22]. Their study sample contained $24 \% \quad(n=359)$ adherent users who completed a 3-month course of therapy with at least $80 \%$ of drug supplied days, $29 \%(n=295)$ partially adherent users with $<80 \%$ of drug supplied days, and 56\% $(n=823)$ non-adherent users, who were prescribed VAR but never filled the claim. In their study, adherent users of VAR were almost twice as likely to achieve sustained smoking cessation compared with non-adherent users, while the quitting rate was similar between partially adherent users and non-adherent users. Consistent with their findings, our results showed that savings in annual SA medical costs offset higher pharmacy costs among adherent users of VAR versus the NRT patch, leading to statistically insignificant differences in annual SA total costs between cohorts; this, however, was not observed in the initiator population, where patients had various levels of adherence. In our study, where data were from various healthcare payers across the US, only a small proportion of patients were identified as adherent users $(18.5 \%$ among VAR initiators and $7.8 \%$ among NRT patch initiators). Thus, findings in our study suggested the need to improve adherence to smoking-cessation therapies among patients treated in US general practices. It is possible that the sample size of the adherent population in our study may not be able to detect statistical significance in the differences between cohorts. Thus, further analysis is needed to confirm the economic benefit of improving adherence in smokingcessation therapies.

This study implemented a two-part regression model to analyze economic outcomes, which can handle the large proportion of patients with zero costs while providing more insights into the utilization process [43]. Coefficients of the regressions (results will be provided upon request) showed that patients in the VAR cohort were more likely to incur any medical costs (SA and all-cause) in Q1 than those in the NRT patch cohort; however, among those with any medical costs, the VAR cohort incurred a lower amount. When stratified by healthcare settings, VAR initiators were more likely to have any outpatient costs but less likely 
to have any inpatient/ER costs (SA and allcause) in Q1, and, given any costs, they had a lower amount of outpatient costs and similar inpatient/ER costs as NRT patch initiators. This implies that VAR initiators may seek more services to monitor smoking cessation during the treatment course (usually 3 months). In Q2-Q4, the VAR cohort had a lower chance of incurring any medical costs and, given costs incurred, a lower amount. Similar patterns were found in the adherent population.

The robustness of our findings was tested through propensity score matching to control for potential confounding. The results from the matched cohorts were consistent with the main analysis: use of VAR versus the NRT patch was associated with lower SA and all-cause medical costs, lower all-cause total costs, and higher SA total costs during the year after therapy initiation. In general, findings in the main analysis hold in the sensitivity analyses despite varying the analytical approach and study population; differences in some cost outcomes between cohorts may become statistically insignificant in the sensitivity analyses due to a smaller sample size.

There was mixed evidence on how using VAR affected SA total costs. In the initiator population, annual SA total costs associated with the use of VAR versus the NRT patch were higher in the main analysis but were similar between matched cohorts with at least 12 months of follow-up; in the adherent population, the difference between cohorts was not statistically significant in the main analysis, but the VAR cohort had higher annual SA total costs than the matched NRT patch cohort with at least 12 months of follow-up. Further analysis is needed to explore this issue.

Comparative studies on smoking-cessation therapies have focused on effectiveness and/or clinical endpoints. Evidence from the US was based on specific smoking-cessation programs or local health centers $[16,19,25]$. Outside of the US, a prospective cohort study, which obtained electronic medical records from 654 general practices across the UK, was conducted to compare long-term effects of VAR versus NRT [44]. Smoking abstinence, mortality, and other clinical endpoints were examined for up to
4 years among more than 200,000 patients prescribed VAR (approximately $32 \%$ ) or NRT in primary settings. Results showed that patients initiating VAR were more likely to be abstinent than those initiating NRT from 3 months to 4 years of follow-up, and the effectiveness of VAR did not change by socioeconomic position [27]. Patients prescribed VAR also had a lower frequency of primary care visits than those on NRT (evaluated every 3 months during the 4 years following initial prescription), but the risks of mortality, hospitalization, or primary care diagnosis of myocardial infarction or COPD were similar between users of the two therapies [18]. Using national US data, our study found that the use of VAR versus the NRT patch was associated with lower outpatient and inpatient/ER costs during the year after therapy initiation. This suggests that the effectiveness of VAR could have a more substantial economic impact in the US than in the UK due to differences in healthcare systems.

This study is subject to several limitations. First, out-of-pocket purchases of over-thecounter (OTC) NRT products are not captured in the claims data. Thus, combination therapy of VAR and OTC NRT cannot be identified. Clinical trials have shown better efficacy of VAR in combination with NRT than VAR alone [45]; however, an analysis of patients who attended tobacco treatment programs in Canada showed a similar abstinence rate among dual VAR and NRT users, dual NRT users, and single NRT users [23]. With mixed results in the literature and unknown patterns of prescription and OTC NRT, the expected real-world impact of the unbalanced missing information is uncertain. Besides the OTC drug use, other unobserved confounding is possible. Second, administrative claims data are primarily collected for purposes other than research, and the analysis is constrained by possible coding errors and missing data. Notably, smoking-cessation counseling outside of claims was not captured. Third, the database contained information from US Commercial and Medicare Supplemental populations, which may not be generalizable to the entire US population of smokers using smokingcessation therapies. Given these limitations, the study results can only be interpreted within the 
context of use of smoking-cessation therapies adjudicated through pharmacy claims, and no casual relationships can be established.

\section{CONCLUSIONS}

Lower medical costs (SA and all-cause) associated with use of VAR, as compared to the NRT patch, were found as early as 3 months after therapy initiation. In the first year after therapy initiation, the savings in medical costs potentially offset the higher pharmacy costs of VAR versus the NRT patch among adherent users. Additionally, the initiation of VAR was associated with lower all-cause total healthcare costs than the NRT patch.

\section{ACKNOWLEDGEMENTS}

Funding. Sponsorship for this study, articleprocessing charges and the open access fees were funded by Pfizer. All authors had full access to the data in this study and take complete responsibility for its integrity and accuracy of the analysis.

Medical Writing and Assistance. Editorial assistance in the preparation of this article was provided by Michael Grossi, an employee of Evidera. Support for this assistance was funded by Pfizer.

Authorship. All named authors meet the International Committee of Medical Journal Editors criteria for authorship for this article, take responsibility for the integrity of the work, and approved this version for publication.

Disclosures. L. Lee is employed by and owns stock in Pfizer. M Bruno is employed by and owns stock in Pfizer. B. Emir is employed by and owns stock in Pfizer. N. Marchant is employed by and owns stock in Pfizer. P. Park is employed by and owns stock in Pfizer. Q. Li is employed by Evidera. S. Shah is employed by Evidera. B. Murphy is employed by Evidera. M. Reynolds is employed by Evidera. Evidera (a division of
PPD) provides consulting and other research services to pharmaceutical, device, and related organizations. In their salaried positions, they work with a variety of companies and organizations, and are precluded from receiving payment or honoraria directly for services rendered. Evidera received funding from Pfizer to participate in the study and for the development of this manuscript.

Compliance with Ethics Guidelines. This article does not contain any studies with human participants or animals performed by any of the authors.

Data Availability. The datasets generated and/or analyzed during the current study are not publicly available due to data license agreement, but are available from the corresponding author on reasonable request.

Open Access. This article is distributed under the terms of the Creative Commons Attribution-NonCommercial 4.0 International License (http://creativecommons.org/licenses/ by-nc/4.0/), which permits any noncommercial use, distribution, and reproduction in any medium, provided you give appropriate credit to the original author(s) and the source, provide a link to the Creative Commons license, and indicate if changes were made.

\section{REFERENCES}

1. Centers for Disease Control and Prevention. Current Cigarette Smoking among Adults in the United States 2016.

2. US Department of Health and Human Services. The health consequences of smoking-50 years of progress: a report of the Surgeon General: full report. Atlanta, GA: US Department of Health and Human Services, Centers for Disease Control and Prevention, National Center for Chronic Disease Prevention and Health, Office on Smoking and Health; 2014.

3. US Department of Health and Human Services. How Tobacco Smoke Causes Disease. The Biology and Behavioral Basis for Smoking- 
Attributable Disease: A report of the Surgeon General. Atlanta, GA, USA; 2010.

4. US Department of Health and Human Services. The health consequences of smoking- 50 years of progress: a report of the Surgeon General: full report 2014.

5. Xu X, Bishop EE, Kennedy SM, Simpson SA, Pechacek TF. Annual healthcare spending attributable to cigarette smoking: an update. Am J Prev Med. 2015;48(3):326-33.

6. Centers for Disease Control and Prevention. Smoking and tobacco use: benefits of quitting. https://www.cdc.gov/tobacco/quit_smoking/how_ to_quit/benefits/index.htm. Accessed 8 Dec 2018.

7. Maciosek MV, Xu X, Butani AL, Pechacek TF. Smoking-attributable medical expenditures by age, sex, and smoking status estimated using a relative risk approach. Prev Med. 2015;77:162-7.

8. Baker CL, Flores NM, Zou KH, Bruno M, Harrison VJ. Benefits of quitting smoking on work productivity and activity impairment in the United States, the European Union and China. International journal of clinical practice. 2017;71(1):e12900.

9. Babb S, Malarcher A, Schauer G, Asman K, Jamal A, Centers for Disease Control and Prevention. Quitting smoking among adults-United States, 2000-2015. Morb Mortality Wkly Rep. 2017;65(52):1457-64.

10. Onor IO, Stirling DL, Williams SR, et al. Clinical effects of cigarette smoking: epidemiologic impact and review of pharmacotherapy options. Int J Environ Res Public Health. 2017;14(10):1147.

11. Fiore MC, Jaén CR, Baker TB, et al. Treating tobacco use and dependence: 2008 update. Rockville: US Department of Health and Human Services; 2008.

12. Cahill K, Stevens S, Lancaster T. Pharmacological treatments for smoking cessation. JAMA. 2014;311(2):193-4.

13. Cahill K, Lindson-Hawley N, Thomas KH, Fanshawe TR, Lancaster T. Nicotine receptor partial agonists for smoking cessation. Cochrane Database Syst Rev. 2016;5:006103.

14. Keating GM, Lyseng-Williamson KA. Varenicline: a pharmacoeconomic review of its use as an aid to smoking cessation. PharmacoEconomics. 2010;28(3):231-54.

15. Halpern MT, Dirani R, Schmier JK. The cost effectiveness of varenicline for smoking cessation. Manag Care Interface. 2007;20(10):18-25.
16. Biazzo LL, Froshaug DB, Harwell TS, et al. Characteristics and abstinence outcomes among tobacco quitline enrollees using varenicline or nicotine replacement therapy. Nicotine Tob Res. 2010;12(6):567-73.

17. Carney G, Maclure M, Taylor S, et al. Effectiveness and safety of smoking cessation pharmacotherapy: a retrospective cohort study. Pharmacoepidemiol Drug Saf. 2016;25(Suppl 3):580.

18. Davies NM, Taylor GMJ, Taylor AE, et al. The effects of prescribing varenicline on two-year health outcomes: an observational cohort study using electronic medical records. Addiction. 2018;113(6):1105-16.

19. Dhelaria RK, Friderici J, Wu K, Gupta E, Khan C, Rothberg MB. Effectiveness of varenicline for smoking cessation at 2 urban academic health centers. Eur J Internal Med. 2012;23(5):461-4.

20. Jung JW, Jeon EJ, Kim JG, et al. Clinical experience of varenicline for smoking cessation. Clin Respir J. 2010;4(4):215-21.

21. Kaduri P, Voci S, Zawertailo L, Chaiton M, McKenzie K, Selby P. Real-world effectiveness of varenicline versus nicotine replacement therapy in patients with and without psychiatric disorders. J Addict Med. 2015;9(3):169-76.

22. Liberman JN, Lichtenfeld MJ, Galaznik A, et al. Adherence to varenicline and associated smoking cessation in a community-based patient setting. J Manag Care Pharm. 2013;19(2):125-31.

23. Okoli CTC, Wiggins A, Fallin-Bennett A, Rayens MK. A retrospective analysis of the comparative effectiveness of smoking cessation medication among individuals with mental illness in community-based mental health and addictions treatment settings. J Psychiatr Ment Health Nurs. 2017;24(8):610-9.

24. Sicras-Mainar A, Diaz-Cerezo S, de Burgoa VS, Navarro-Artieda R. Cost and clinical consequences of smoking cessation in outpatients after cardiovascular disease: a retrospective cohort study. Clinicoecon Outcomes Res. 2013;5:419-27.

25. Steinberg MB, Bover MT, Richardson DL, Schmelzer AC, Williams JM, Foulds J. Abstinence and psychological distress in co-morbid smokers using various pharmacotherapies. Drug Alcohol Depend. 2011;114(1):77-81.

26. Tannverdi H, Altuntas M, Demir O, Afşar B, Çelikiz M. Success rates of pharmacological therapies used for smoking cessation and factors that affect smoking cessation rates. Eur J Gen Med. 2015;12(2):125-30. 
27. Taylor GM, Taylor AE, Thomas $\mathrm{KH}$, et al. The effectiveness of varenicline versus nicotine replacement therapy on long-term smoking cessation in primary care: a prospective cohort study of electronic medical records. Int $\mathrm{J}$ Epidemiol. 2017;46(6):1948-57.

28. Wu I, Chen $\mathrm{H}$, Bordnick $\mathrm{P}$, et al. Comparative effectiveness of smoking cessation medications among schizophrenic smokers. Value Health. 2016;19(3):A119-20.

29. Yang M, Chen H, Johnson ML, et al. Comparison of diabetes risk following smoking cessation treatment using varenicline versus bupropion among obese smokers. Subst Use Misuse. 2015;50(13):1628-37.

30. Yilmazel Ucar E, Araz O, Yilmaz N, et al. Effectiveness of pharmacologic therapies on smoking cessation success: three years results of a smoking cessation clinic. Multidiscip Respir Med. 2014;9(1):9.

31. Brose LS, West R, Stapleton JA. Comparison of the effectiveness of varenicline and combination nicotine replacement therapy for smoking cessation in clinical practice. Mayo Clin Proc. 2013;88(3):226-33.

32. Chang PY, Lo PC, Chang HC, Hsueh KC, Tsai YW. Comparative effectiveness of smoking cessation medications: a national prospective cohort From Taiwan. PLoS ONE. 2016;11(11):e0166992.

33. Cartmell KB, Dooley M, Mueller M, et al. Effect of an evidence-based inpatient tobacco dependence treatment service on 30-, 90-, and 180-day hospital readmission rates. Med Care. 2018;56(4):358-63.

34. Mullen KA, Manuel DG, Hawken SJ, et al. Effectiveness of a hospital-initiated smoking cessation programme: 2-year health and healthcare outcomes. Tob Control. 2017;26(3):293-9.

35. Land T, Rigotti N, Levy D, et al. A longitudinal study of medicaid coverage for tobacco dependence treatments in Massachusetts and associated decreases in hospitalizations for cardiovascular disease. PLoS Med. 2010;7(12):e10000375.

36. Cartmell KB, Dismuke CE, Dooley M, et al. Effect of an evidence-based inpatient tobacco dependence treatment service on 1-year postdischarge health care costs. Med Care. 2018;56(10):883-9.
37. Sicras-Mainar A, Rejas-Gutierrez J, Navarro-Artieda $\mathrm{R}$, Ibanez-Nolla J. The effect of quitting smoking on costs and healthcare utilization in patients with chronic obstructive pulmonary disease: a comparison of current smokers versus ex-smokers in routine clinical practice. Lung. 2014;192(4):505-18.

38. Charlson ME, Charlson RE, Peterson JC, Marinopoulos SS, Briggs WM, Hollenberg JP. The Charlson comorbidity index is adapted to predict costs of chronic disease in primary care patients. J Clin Epidemiol. 2008;61(12):1234-40.

39. Afifi AA, Kotlerman JB, Ettner SL, Cowan M. Methods for improving regression analysis for skewed continuous or counted responses. Annu Rev Public Health. 2007;28:95-111.

40. Liang KY, Zeger S. Longitudinal data analysis using generalized linear models. Biometrika. 1986;73(1):13-22.

41. Austin PC. Balance diagnostics for comparing the distribution of baseline covariates between treatment groups in propensity-score matched samples. Stat Med. 2009;28(25):3083-107.

42. Anthenelli RM, Benowitz NL, West R, et al. Neuropsychiatric safety and efficacy of varenicline, bupropion, and nicotine patch in smokers with and without psychiatric disorders (EAGLES): a doubleblind, randomised, placebo-controlled clinical trial. Lancet. 2016;387(10037):2507-20.

43. Diehr P, Yanez D, Ash A, Hornbrook M, Lin DY. Methods for analyzing health care utilization and costs. Annu Rev Public Health. 1999;20:125-44.

44. Davies NM, Taylor G, Taylor AE, et al. What are the effects of varenicline compared with nicotine replacement therapy on long-term smoking cessation and clinically important outcomes? Protocol for a prospective cohort study. BMJ Open. 2015;5(11):e009665.

45. Chang PH, Chiang CH, Ho WC, Wu PZ, Tsai JS, Guo FR. Combination therapy of varenicline with nicotine replacement therapy is better than varenicline alone: a systematic review and meta-analysis of randomized controlled trials. BMC Public Health. 2015;15:689. 